Trauma Berufskrankh 2008 · 10

[Suppl 3]:411-415

DOI 10.1007/s10039-007-1346-9

Online publiziert: 26. Januar 2008

(c) Springer Medizin Verlag 2008

\author{
U. Römer \\ BG-Unfallklinik, Tübingen
}

\title{
Ergotherapie bei Querschnittlähmung
}

traplegikern zunächst mit der Ausbildung der Funktionshand beginnt.

\section{Ausbildung der Funktionshand} enten lassen sich wie folgt formulieren:

- Erlangung größtmöglicher Selbstständigkeit durch Nutzung verbliebener Funktionen

- Freie Lebensgestaltung im Rahmen der funktionellen Möglichkeiten

- Bewältigung des Alltags in maximaler Unabhängigkeit von Dritten

Daraus ergibt sich auch der Begriff „funktionsorientierte Behandlung“, die bei Te-
Sie setzt bereits in der Frühphase auf der Intensivstation ein: Die Finger werden in Funktionshandstellung fixiert [MCP (Metakarpophalangealgelenk), PIP (proximales Interphalangealgelenk) in $90^{\circ}$ Flexion, DIP (distales Interphalangealgelenk) in $\mathrm{O}^{\circ}$, - Abb. 1a] und das Handgelenk in $30^{\circ}$ Extension gelagert ( $\mathbf{A b b}$. 1b). Dies bewirkt die gewünschte Verkürzung der Fingerflexoren und vermeidet gleichzeitig die Verkürzung der Fingerextensoren sowie der Kollateralbänder von Hand- und Fingergelenken. Es kommt zum Tenodeseneffekt (Sehnenfunktion), der zum Greifen ausgenutzt wird, und somit zur aktiven Funktionshand: Bei aktiver Flexion (sub C6) des Handgelenks resultiert eine passive Extension der Finger (- Abb. 2a), bei aktiver Extension des Handgelenks eine passive Flexion der Finger (• Abb. 2b).

Bei einer Läsionshöhe sub $C_{5}$ sprechen wir von einer passiven Funktions-
Abb. 1 Funktionshand, a in Funktionshandstellung fixierte Finger, $\mathbf{b}$ optimale Lagerung der Hand

Abb. 2 Aktive Funktionshand, a passive Extension der Finger bei aktiver Flexion des Handgelenks, b passive Flexion der Finger bei aktiver Extension des Handgelenks
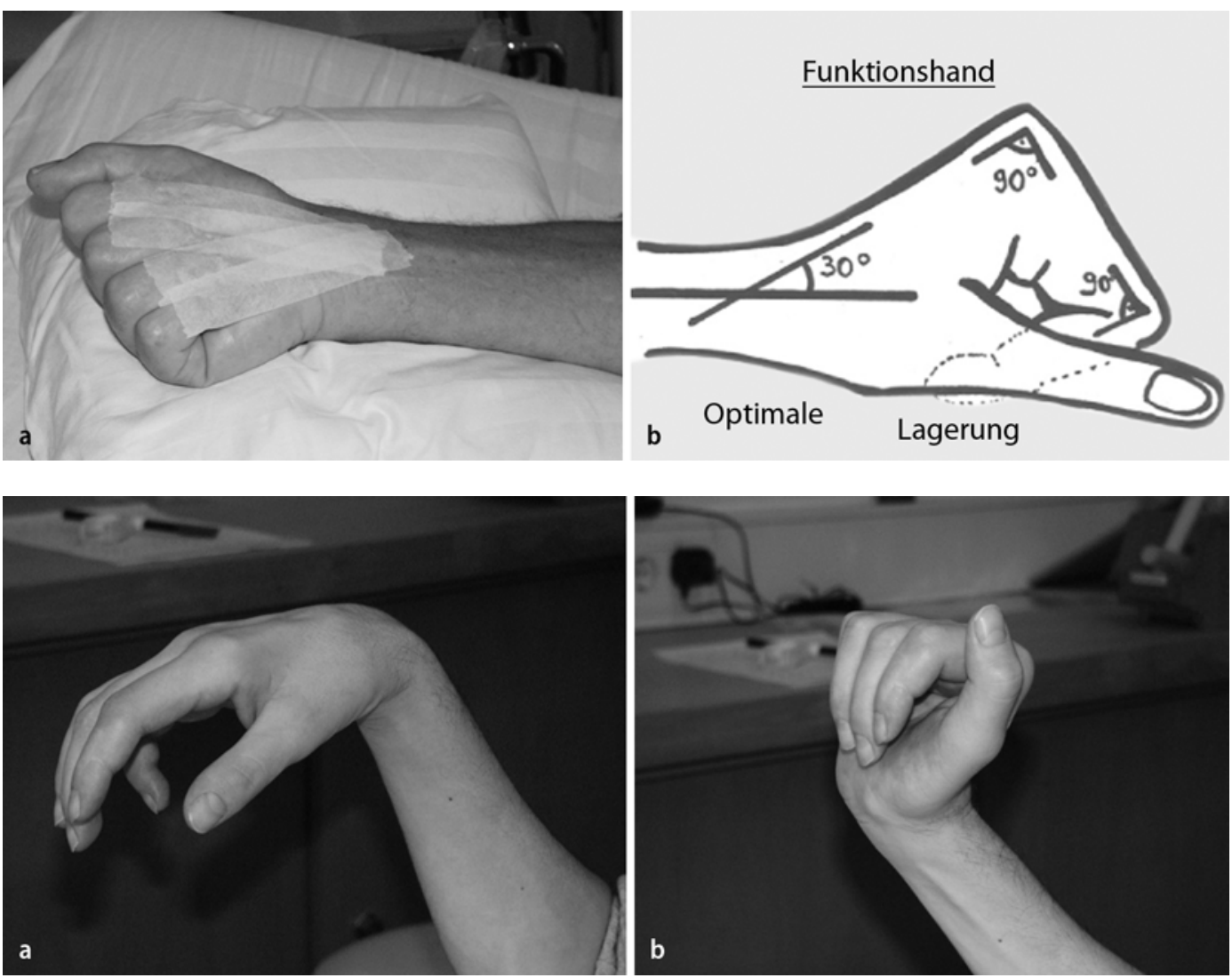


\section{Verletzungen der Wirbelsäule}

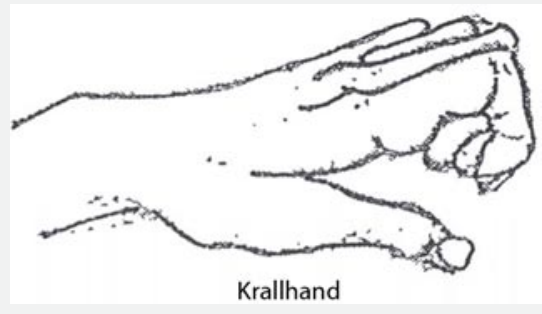

Abb. $3 \Delta$ Krallhand
Abb. 6 Individuell adaptierte PC-Maus
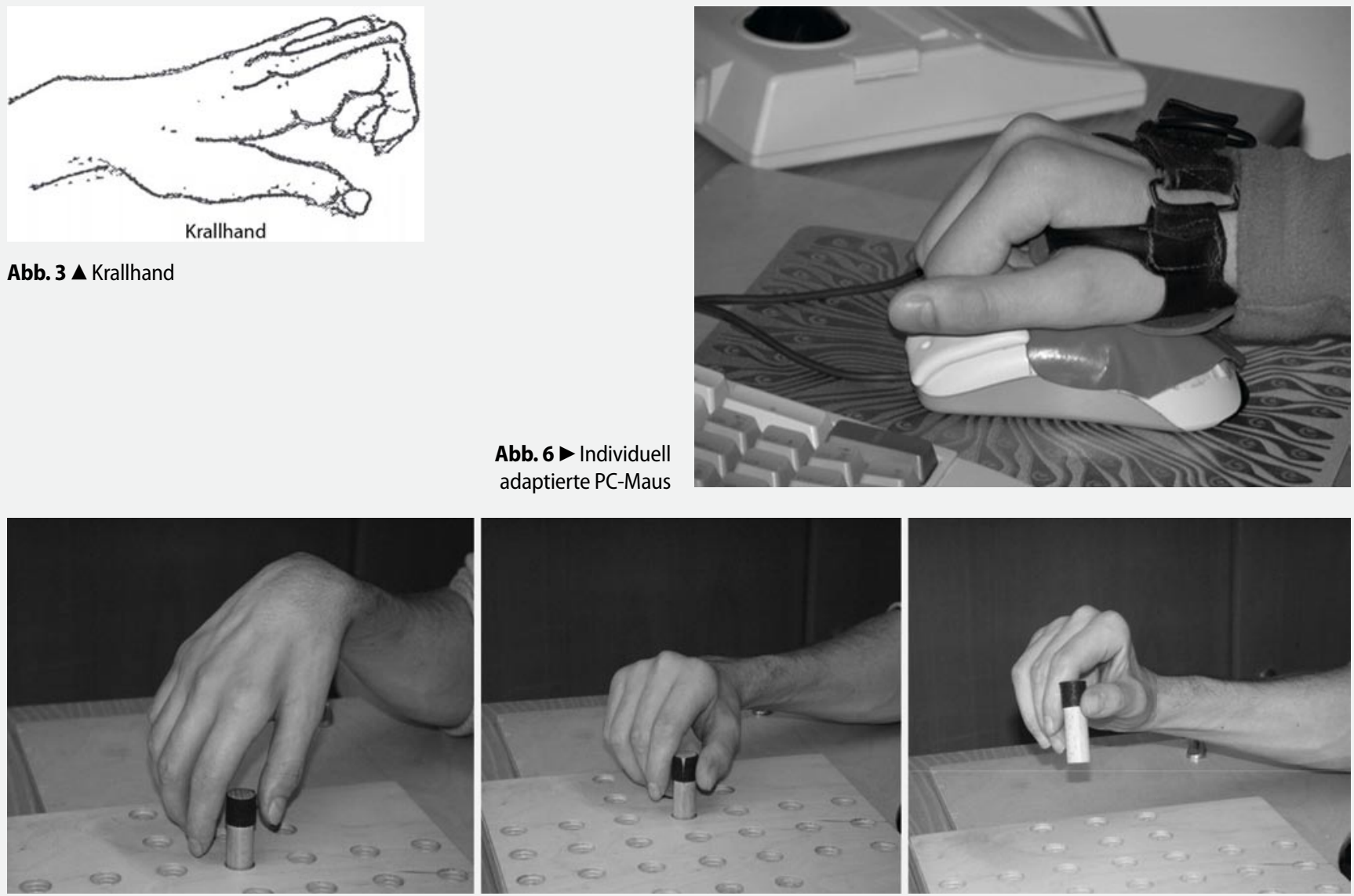

Abb. $4 \Delta$ Aktive Funktionshand, Tenodeseneffekt

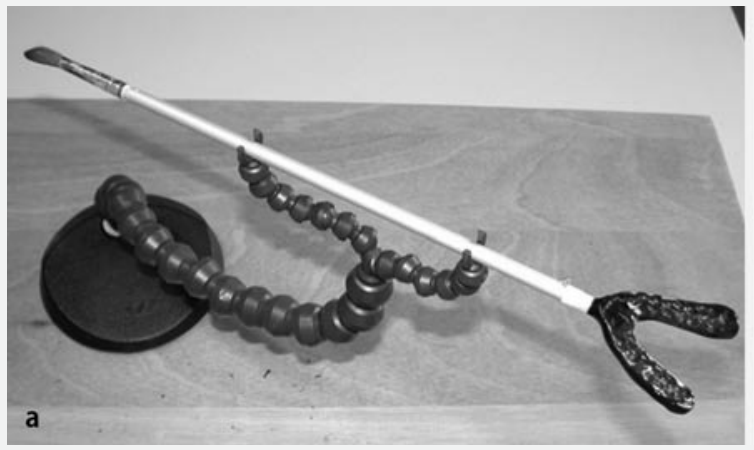

b

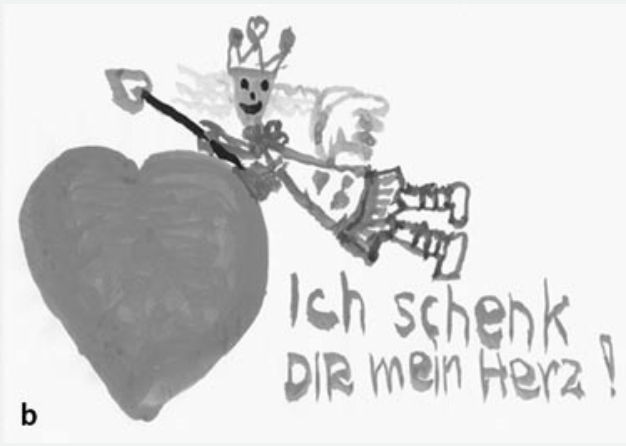

Abb. $5<$ a Mundstab, b Mundstabarbeit zum Valentinstag hand. Die passive Flexion bzw. Extension des Handgelenks werden durch die Probzw. Supination des Unterarms erreicht.

Wird nicht gelagert, entstehen tendenziell Handformen, die im Gegensatz zur Funktionshand kaum Möglichkeiten für einhändiges Greifen bieten (• Abb. 3). Für die Stützfunktionen ist eine gute Streckung des Ellenbogens wichtig. Diese darf somit bei der Lagerung während der Liegephase nicht vernachlässigt werden.

Um Kontrakturen in den Finger- und Handgelenken vorzubeugen, werden diese mindestens 2-mal täglich mobilisiert. Dabei ist zu beachten, dass die MCP, PIP und DIP nur bei gleichzeitiger Handgelenkflexion extendiert bzw. bei -extension flektiert werden.

Oft ist der Einsatz von Lagerungsschienen zur Kontrakturprophylaxe sinnvoll.

Zur Unterstützung des Handgelenks bei fehlender Extensorfunktion (sub $\mathrm{C}_{5}$ ) wird meist eine Handgelenkmanschette eingesetzt.

\section{Funktionelle Therapie}

Durch eine intensive Handfunktionstherapie wird der Einsatz der Funktionshand (• Abb.4) in täglichen Verrichtungen maximal trainiert, und vielfältige Griffe werden wiedererlernt.

Neben dem Funktionshandtraining sind Bewegungsschulung, Kräftigung und Gelenkmobilisation der gesamten oberen Extremität ein wichtiger Bestandteil der ergotherapeutischen Behandlung. Zum Einsatz kommen u. a. Behandlungstechniken wie Manuelle Therapie und Perfetti-Übungen.

Mit speziellen Werkzeughandschuhen kann der Patient in der Holzwerkstatt systematisch Kraft im Rahmen seiner Möglichkeiten auftrainieren. Bei Patienten mit reduzierter Feinmotorik (sub C7, sub C8) 
kommen z. B. Flechtarbeiten mit Peddigrohr oder adaptierte Spiele zum Einsatz.

Für Patienten ohne jegliche Armfunktion besteht die Möglichkeit der „Mundstabarbeit“. Die Mundstäbe (• Abb. 5a) werden vom Kieferorthopäden anhand des Gebissabdrucks gefertigt. Am mundfernen Ende können Pinsel, Stifte oder Magneten befestigt werden, und der Patient kann Schreiben bzw. Malen (- Abb.5b) lernen und kräftigt dabei u. a. auch seine Halsmuskulatur (Atemhilfsmuskulatur).

\section{Activities of daily living (ADL)}

Ziele des ADL-Trainings sind:

- Erlernen von Basisaktivitäten für zu Hause, die Freizeit und als Voraussetzung für Mobilität

- Erlernen und Anwenden von Trickbewegungen, Ersatzfunktionen sowie Kompensationsmechanismen

- Hilfsmittelberatung und -versorgung: „so viel wie nötig, so wenig wie möglich,

\section{Basisaktivitäten}

Zum ADL-Training gehören:

- Körperpflege: Rasieren, Kämmen, Zähneputzen (ggf. mit Hilfsmitteln wie Zahnbürsten- bzw. Rasierapparathalterung)

- Anziehtraining im Bett und Rollstuhl, ggf. mit adaptierten Kleidungsverschlüssen, Greifzange, Strumpfanziehhilfe

- Esstraining (ggf. Mittelhandspange zur Aufnahme von Gabel und Löffel, adaptierte Messer, usw.): Aufschneiden, Bestreichen, Greifen und zum Mund Führen eines Brötchens, Eingießen von Getränken, zum Mund Führen eines Bechers/Glasses

- Transfer: vom Rollstuhl ins Auto und zurück, mit oder ohne Rutschbrett, Ein- und Ausladen des Rollstuhls, Transfer Rollstuhl - Badewanne Rollstuhl und Anleitung der Angehörigen

- Teilhabe am öffentlichen Leben, z. B. Umgang mit Geld: Öffnen der Geldbörse, Herausnehmen von Münzen und Scheinen, Umgang mit Kassenautomaten

Trauma Berufskrankh 2008 10 [Suppl 3]:411-415 DOI 10.1007/s10039-007-1346-9

(c) Springer Medizin Verlag 2008

\section{U. Römer \\ Ergotherapie bei Querschnittlähmung}

\section{Zusammenfassung}

In Deutschland sind jedes Jahr etwa 1000 Menschen neu von einer Querschnittlähmung betroffen. Häufigste Ursache sind Unfälle mit Frakturen im Bereich der Wirbelsäule. Die Läsionshöhe wird durch das letzte noch intakte Rückenmarksegment definiert. Die Diagnose "Querschnitt unterhalb C6" (sub (6) bedeutet, dass das letzte noch intakte Segment C6 ist. Interessant ist die Bandbreite des Selbstständigkeitspotenzials bei Tetraplegikern. Während Betroffene mit Läsionen zwischen $\mathrm{C} 1$ und $\mathrm{C} 3$ auf Dauerbeatmung angewiesen sind, Aktivitäten am Computer mit elektronischen Hilfsmitteln ausführen und den Elektrorollstuhl mit Mundbzw. Kinnsteuerung fahren, kann der Betrof- fene mit einer Läsion sub C6 schon eine weitgehende Selbstständigkeit in den Alltagsverrichtungen in angepasster Umgebung unter stark erhöhtem Zeit- und Kraftaufwand erreichen. Bei Läsionen sub C7 und C8 erweitert sich das Selbstständigkeitspotenzial aufgrund der vorhandenen Fingerextension bzw. der Fingerfunktion mit reduzierter Feinmotorik. Daraus ergibt sich ein vielfältiges und anspruchsvolles Arbeitsgebiet für Ergotherapeuten/-innen.

\section{Schlüsselwörter}

Querschnittlähmung · Läsionshöhe . Selbstständigkeitspotenzial · Hilfsmittel · Ergotherapie

\section{Occupational therapy for paraplegia}

\section{Abstract}

Every year about 1,000 people in Germany become paraplegic, mainly because of accidents involving a fracture in the spinal column. The resulting level of paralysis is defined by the last intact spinal segment. For example, a diagnosis "paralysis below C6" means that the last intact spinal segment is C6. In this context, it is very interesting to look at the spectrum of independence of individuals with the diagnosis of quadriplegia. While those with lesions between $\mathrm{C} 1$ and C3 depend on continuous artificial respiration, can do computer activities only with electronic aids, and drive electric wheelchairs using mouth or chin control, individuals with lesions below $\mathrm{C} 6$ can achieve (with a strong- ly increased expenditure of time and energy) much more autonomy in everyday life in an adapted environment. For those with transverse lesions below $\mathrm{C} 7$ and $\mathrm{C} 8$, further increases in independence are possible because of finger extension and finger function with reduced fine motor skills. Therefore, persons with transverse lesions of the spinal cord represent a multifaceted and challenging area of work for occupational therapists.

\section{Keywords}

Paraplegia - Level of paralysis . Autonomy in everyday life - Accessibility aids . Occupational therapy 


\section{Verletzungen der Wirbelsäule}

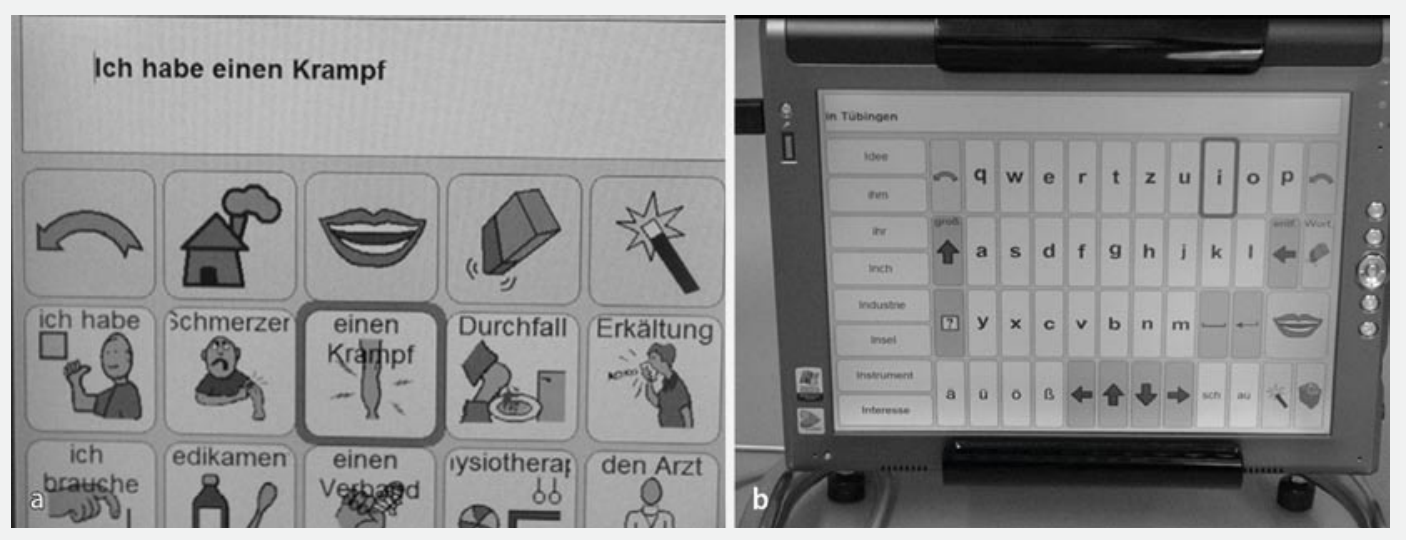

Abb. 7 Kommunikation über PC-Programme mit Augensteuerung

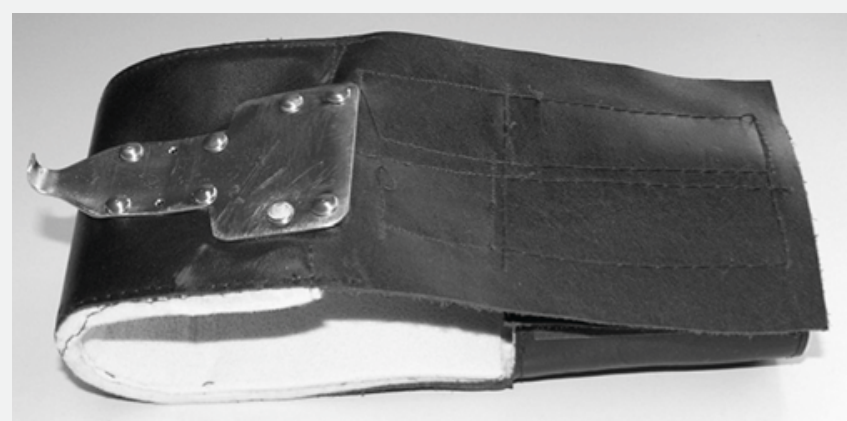

Abb. $8 \Delta$ Individuell angepasster Bogenschießhandschuh

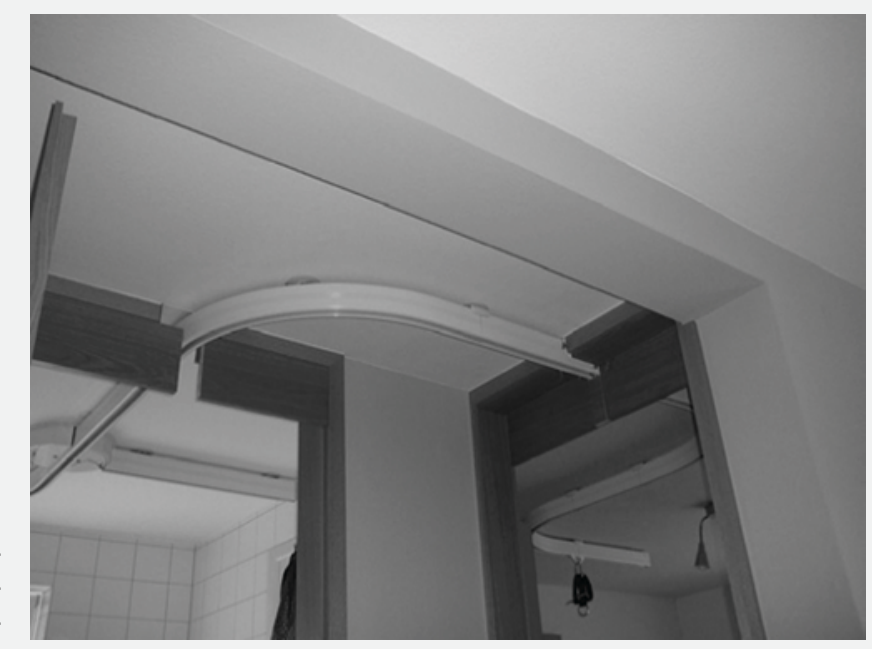

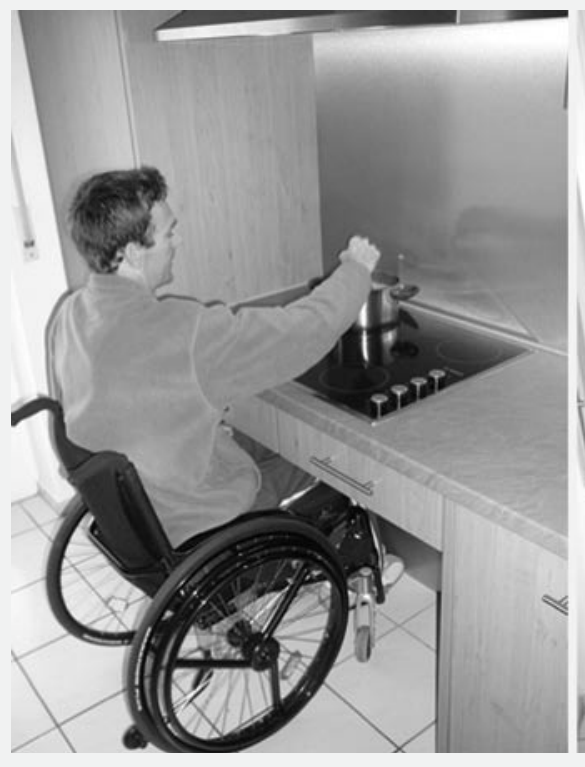

- Bei Bedarf Küchen- und Haushaltstraining

- usw.

\section{Kommunikation}

Hier sind aufzuführen:

Abb. 9 Deckenlifter vom Bad über den Flur ins Schlafzimmer

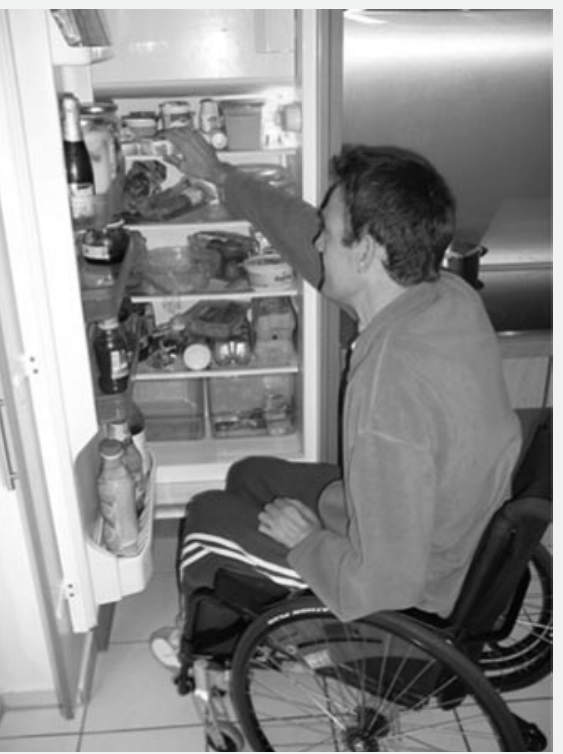

- Schreibtraining, v. a. auch der Unterschrift, mit spezieller Stifthalterung bei fehlender Fingerfunktion

- Telefonieren mit entsprechenden Halterungen an Telefonhörer oder Handy

- Umblättern von Buchseiten und Zeitschriften, evtl. mit elektrischen Blätterhilfen
Abb. $10 \varangle$ Küchenumgestaltung, a unterfahrbare Arbeitsplatte, $\mathbf{b}$ Schränke in Greifhöhe

- PC-Training, z. B. mit Tipphämmerchen oder individuell adaptierter herkömmlicher Maus (• Abb. 6)

- Versorgung mit speziellem Zubehör: programmierbare Bildschirmtastatur, Mundmaus, Sprachsteuerung usw. 
Für beatmete Patienten mit fehlender aktiver Sprachproduktion gibt es z. B. einen PC mit Augensteuerung. Dazu wird zunächst ein Sensor auf den Augenabstand kalibriert. Der Betroffene kann nun mit den Augen das gewünschte Wort/Bild ansteuern und durch Augenzwinkern bestätigen. Die so ausgewählten Symbole/ Worte/Sätze werden dann über das Symbol „sprechen“, durch ein weiteres Augenzwinkern aktiviert und über die Sprachausgabe des PC ausgegeben ( $\bullet$ Abb. 7).

\section{Hilfsmittelberatung, -versorgung, -herstellung}

Neben den oben erwähnten Halterungen, die wir individuell für jeden Patienten aus PVC herstellen, fertigen wir aus Leder so genannte Rollstuhlhandschuhe an. Diese werden im Bereich der Handinnenfläche mit einer rutschfesten Auflage versehen, damit die Kraft beim Rollstuhlfahren optimal auf die Greifreifen übertragen wird.

Je nach Läsionshöhe des tetraplegischen Patienten erfolgt eine entsprechende Anpassung zur Steuerung des Elektrorollstuhls mit Mund-, Kinn- oder Handsteuerung.

Auch Umfeldkontrollsysteme zur Steuerung von Notruf, TV, Licht, Radio, Türen, Telefon usw. werden erprobt.

Tetraplegische Patienten, die an Aktivitäten im Bereich Sport teilnehmen, werden mit individuell angepassten Tischtennis- bzw. Bogenschießhandschuhen (- Abb. 8) versorgt. Mit entsprechend adaptierten Sportgeräten, wie dem Handfahrrad mit spezieller Griffadaption und zuschaltbarem elektrischem Antrieb können neue Perspektiven gefunden werden.

Besonders wichtig für viele ist die Mobilität im eigenen Auto. Fahrzeugumrüster entwickelten dazu eine Vielfalt von Möglichkeiten, und spezielle Fahrschulen bieten Schulungen auf adaptierten Fahrzeugen an.

\section{Wohnberatung}

Um als Rollstuhlfahrer in die bestehende Wohnung zurückkehren zu können, sollte diese möglichst entsprechend angepasst werden:
- Zugang zum Haus, zur Wohnung (evtl. Ausstattung mit Lifter und Rampen)

- Treppen innerhalb des Hauses: Überwindung mit mobilen Treppenraupen für Selbstfahrer oder Begleitpersonen, Überwindung mit fest installierten Treppenliftern

- Ausreichende Türbreiten

- Zugang zum Bad, zur Dusche (ebenerdig), Toilette (überfahrbar mit Dusch- bzw. Toilettenstuhl)

- Schlafzimmer: ausreichend Platz für den Rollstuhl neben dem Bett (evtl. Transfer ins Bett mit Deckenlifter,

- Abb. 9)

- Küche: unterfahrbare Arbeitsplatte, Schränke in Greifhöhe (• Abb. 10)

Die Beratung erfolgt anhand der mitgebrachten Pläne bzw. gemäß DIN 18025 für Rollstuhlbenutzer und barrierefreie Wohnungen.

\section{Kommentar}

Es bleibt anzumerken, dass eine gute Akutbehandlung bei Querschnittlähmung nur in einem gut funktionierenden interdisziplinären Team zu erreichen ist. Tägliche kurze Rückmeldungen über Vorkommnisse des vergangenen Tages, regelmäßige interdisziplinäre Besprechungen zum Behandlungsverlauf und frühzeitige Einbeziehung der Angehörigen helfen, die gesetzten Ziele sowohl von Seiten der Klinik als auch des Betroffenen zu erreichen.

\section{Korrespondenzadresse}

\section{U. Römer, Ltd. Ergotherapeutin}

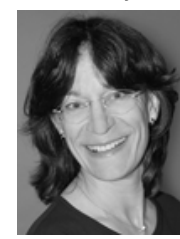

BG-Unfallklinik Tübingen

Scharrenbergsgtraße 95,

72076 Tübingen

uroemer@bgu-tuebingen.de

Interessenkonflikt. Der korrespondierende Autor gibt an, dass kein Interessenkonflikt besteht.

\section{Literatur}

1. Joss R (2004) Kursscript: Funktionsorientierte Behandlung der oberen Extremitäten bei Tetraplegie. Bern 2004

2. Zäch GA, Koch HG (Hrsg) (2006) Paraplegie Ganzheitliche Rehabilitation. Karger, Basel 